\title{
EFFECT OF CHANGE OF KILOVOLTAGE AND VOXEL SIZE ON 3D VOLUME MEASUREMENT USING CONE BEAM COMPUTED TOMOGRAPHY
}

\author{
Mohamed Omar Elboraey
}

\begin{abstract}
Purpose: The aim of this study is to study the impact of change in kilovoltage (KV) or voxel size on extracted 3D radiographic volume measurement of different sizes of endodontic files and to determine the optimal exposure parameters for volume measurement in CBCT.

Methods: Eight different endodontic $\mathrm{k}$ files were radiographically exposed at three different kilovoltages using CBCT and divided into three groups, group $1(80 \mathrm{KV})$, group $2(60 \mathrm{KV})$, and group $3(50 \mathrm{KV})$, then exposed to two different voxel sizes, and divided into two groups, group a $(300 \mu \mathrm{m})$ and group $\mathrm{b}(180 \mu \mathrm{m})$. The 3D radiographic volume and the actual files volumes were measured using ITK-SNAP software and water displacement method respectively.

Finding: Regarding changes in the kilovoltages, no significant difference $(p>0.05)$ was reported between the mean of the 3D radiographic volume of groups 3 and 2 with the mean of actual volume with a mean difference of $1.1 \pm 7.7 \mathrm{~mm} 3$ and $2.6 \pm 9.6 \mathrm{~mm} 3$ respectively. The results for voxel size change, showed no significant difference between the mean of the 3D radiographic volume of group (b) and actual volume group with a mean difference of $1.9 \pm 7.8 \mathrm{~mm}^{3}$. In the other hand, the difference between the mean of volume measurement at group (a) and actual group became significant with a mean difference of $10.5 \pm 5.7 \mathrm{~mm}^{3}$ was found.
\end{abstract}

Conclusion: it concluded that both changes in kilovoltage and voxel size parameters affect significantly the $3 \mathrm{D}$ volume measurement and reconstruction.

\section{INTRODUCTION}

Diagnostic imaging as Magnetic resonance imaging (MRI), computational tomography (CT), Cone-beam computed tomography (CBCT), proton emission tomography (PET), and ultrasound are of great important not only to diagnose disease but also to plan and guide surgical interventions, monitoring disease progression and the response to treatment.

Cone-beam computed tomography $(\mathrm{CBCT})$ is a widely used modality for radiographic examination of bone at the head and neck region, showing high accuracy and the provided programs with CBCT

* Lecturer of Oral Medicine, Periodontology, Oral Diagnosis and Radiology Department, Faculty of Dentistry, Tanta University, Tanta, Egypt. 
allow easy, rapid radiographic interpretation ${ }^{[1]}$. Recently CBCT was widely replace conventional computed tomography (CT) due to the newly high advances in image quality that become very close to CT images, lower exposure doses, rapid and easy exposure for the patient and easy images interpretation and planning thanks to many available interpretation software ${ }^{[2],[3]}$.

Increasingly, three dimensions (3D) radiographic modalities can be used for extraction of accurate quantitative measures such as 3D volume measurement and reconstruction. For example, MRI can be used to estimate the risk of tumor recurrence in individual patients as quantitative measures of tumor size and shape, intensity heterogeneity and the dynamics of contrast agent uptake can be statistically analysed using a group of digital software to assign the patient individual risk to tumor recurrence and to help for assessment for the the optimal treatment strategy for that patient ${ }^{[4]}$.

Gaining 3D reconstruction models of teeth or bone from CBCT data is becoming increasingly more important in dentistry, as it can used for constructing individual orthodontic appliances, and evaluate treatment results, ${ }^{[5]}$, designing a custom-made implant and 3D implant surgical guide ${ }^{[6,7]}$ and simulate and navigate an oral and maxillofacial surgery ${ }^{[8]}$, so the inaccuracy in CBCT volumetric reconstruction may have important clinical influence, as the inaccuracy in $3 \mathrm{D}$ volumetric reconstruction and measurement will create an unavoidable error in landmark positioning and influence creation of the bone-supported guide template ${ }^{[9]}$.

The accuracy of the 3D reconstruction and 3D volumetric measurements are affected by scanning system, field of view (FOV), examined objects, exposure time, tube voltage and amperage, Hounsfield unit (HU) threshold of segmentation and also spatial resolution defined by the voxel size ${ }^{[10]}$. this effect can lead not only to inaccurate results of the dif- ferent clinical studies but also clinical applications as a reconstructed 3D surgical stent for an implant, $3 \mathrm{D}$ reconstruction teeth or bone model for the orthodontic treatment plan, constructed individual appliances, and evaluated treatment results..$^{[1]}$

Voxel size is one of the critical parameters that influence the volumetric measurement of 3D-reconstructed jaws. Voxel size is the minimum unit of digital data segmentation in threedimensional space, similar in concept to pixels in two-dimensional space. It is of paramount importance in terms of scanning and reconstruction times, as well as the quality of CBCT images ${ }^{[12]}$.

Several programs can be used for 3D volume measurement using Digital Imaging and Communications in Medicine (DICOM) files from CBCT, CT, and MRI, which has become widely used in different dental research as a reliable, reproducible, and valid method for radiographic assessment ${ }^{[13,14]}$.

ITK-SNAP software is a friendly-use, costeffective, and reliable package for measuring radiographic volumes by importing DICOM files from different imaging modalities as MRI, CT, CBCT and ultrasonography as tested for measuring nasopharyngeal volumes and the dimension of the bony defect as in patients with cleft palate before alveolar bone grafting to reduce unnecessary morbidity of the donor site as a result of unnecessary over-harvesting. ${ }^{[15]}$

So, this study was aimed to evaluate the effect of changes in exposure parameters on $3 \mathrm{D}$ volume measurements of different sizes of $k$ endodontic files and to determine the optimal exposure parameters for volume measurement in CBCT.

\section{Study design}

The study sample used, 8 different sizes of $\mathrm{K}$ endodontic files* $(10,15,20,30,55,60,70$ and 80) were inserted into wax stent parallel to each other

\footnotetext{
* Dentsply Sirona, USA.
} 
and exposed to different exposure parameters.

The block was radiographically exposed using CS9300 Carestream* CBCT machine, at Collage of Dentistry, Taibah University, Kingdom of Saudi Arabia. The study was ethically approved by research ethics committee, Collage of Dentistry, Taibah university with reference No, TUCDEEC/07112020/MOELBORAEY.

The files used in this study were new, not used before. The file stopper was located at $\mathrm{D}_{16}$ point of the file (16 $\mathrm{mm}$ from the file tip) as a fixed reference point for all files. All used files were intact, not broken free from any bindings or previous curvature.

The block was exposed to different radiation parameters and divided into groups as the following:

1) Changes in kilovoltages (KV).

Three different KV will be used in this study.

Group (1) (80KV, 300 VOXAL, $4 \mathrm{Ma}$ and 8.01sec)

Group (2) (60KV,300 VOXAL, 4 Ma and 8.01sec)

Group (3) (50 Kv, 300 VOXAL, 4 Ma and 8.01 sec)

Group (actual), represent the data for actual volume measurement.

2) Changes in voxel size.
Two different voxel sizes used in this study.

Group (a) (300 VOXAL, $4 \mathrm{Ma}, 80 \mathrm{KV}$ and $8.01 \mathrm{sec}$ )

Group (b) (180 VOXAL, 4 ma, $80 \mathrm{KV}$ and $8.01 \mathrm{sec}$ )

Group (actual), represent the data for actual volume measurement.

\section{Actual volume measurement:}

- Actual endodontic files volumes were measured from $\mathrm{D}_{0}$ (at file tip) to $\mathrm{D}_{16}$ using the water displacement volume measurement method. A cylindrical tube with fixed diameter was filled with coloured water to a fixed mark, then the file length from $\mathrm{D}_{0}$ to $\mathrm{D}_{16}$ was cut off and then inserted to the tube, the level of water displacement was demarcated and then the displacement height (h) was measured. Using the following equation $V=\pi r^{2}(x) h$ as $v$ (volume), $r$ (tube diameter) and $h$ (height of displacement), the actual file volume was measured ${ }^{[16]}$.

\section{D radiographic volume measurement:}

- 3D radiographic volume of endodontic files was measured from $\mathrm{D}_{0}$ to $\mathrm{D}_{16}$ using ITKSNAP software** The radiographic volume measurements were done by a single expert examiner, using the same version of ITK-SNAP

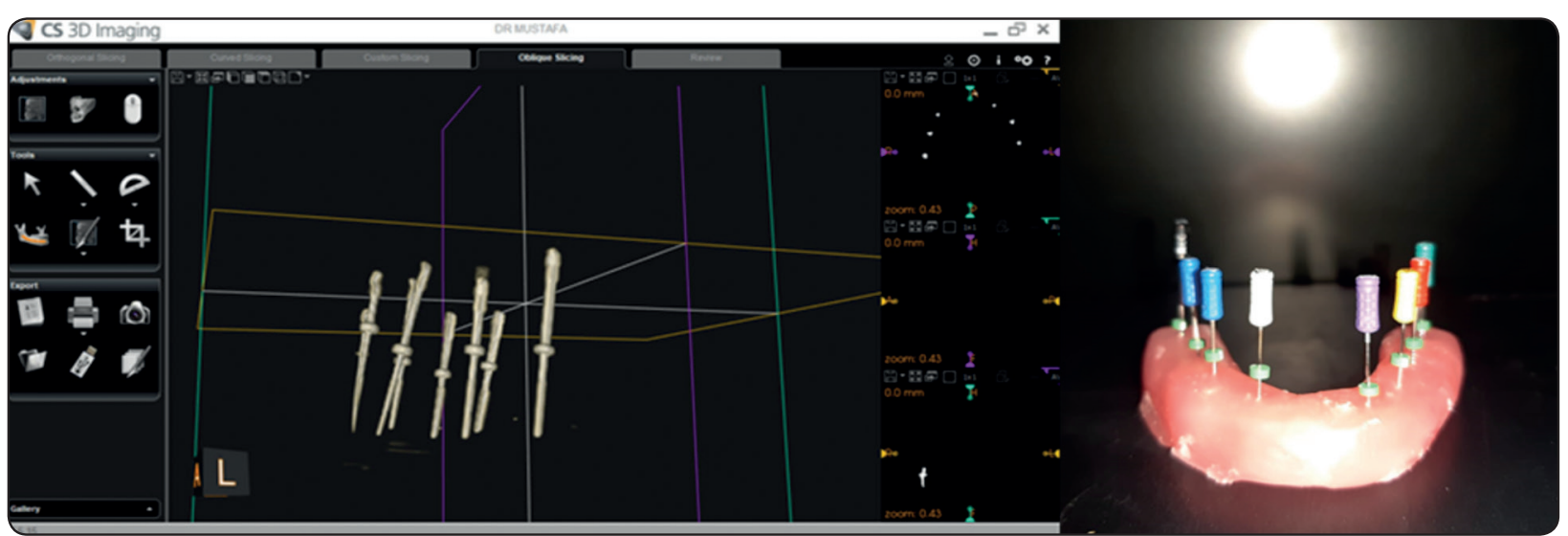

Fig. (1): Eight endodontic files at wax block and after radiographic exposure using CS9300 Carestream CBCT machine.

\footnotetext{
* Carestream Dental, Carestream Health, Inc, USA

** ITK-SNAP version 3.8.0, Supported by the U.S. National Institute of Biomedical Imaging and Bio-Engineering.
} 
software and the same windows operating system with fixed screen resolution.

- The Dicom files were inserted to ITK-SNAP software, then manual expert assessment of each file from $\mathrm{D}_{0}$ to $\mathrm{D}_{16}$ was done using the axial view. The file pixel at each slice was identified and measured slice by slice depending also on artificial intelligent option of isotropic voxel identification. Re-evaluation and recheck from sagittal and coronal views were done for accurate identification.

- Automatic volume measurement was calculated using volume and statistics option of ITKSNAP.

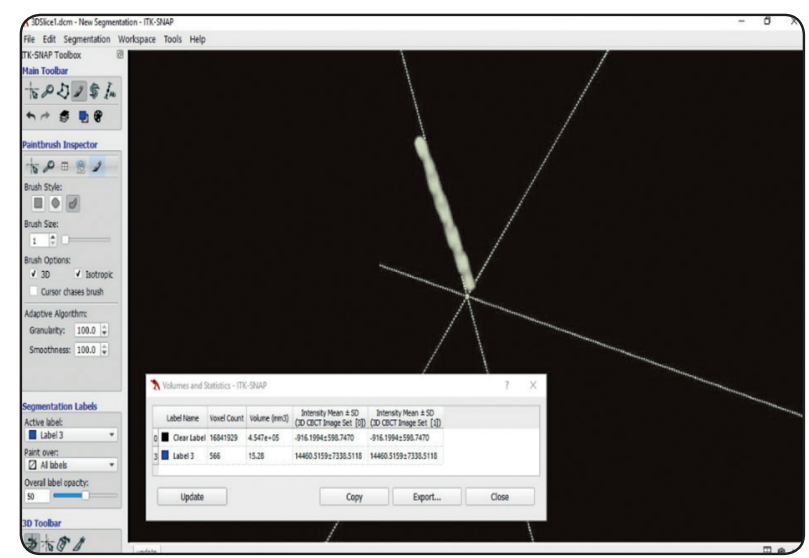

Fig. (2): 3D volume measurement of $k$ endodontic file using ITK-SNAP software.

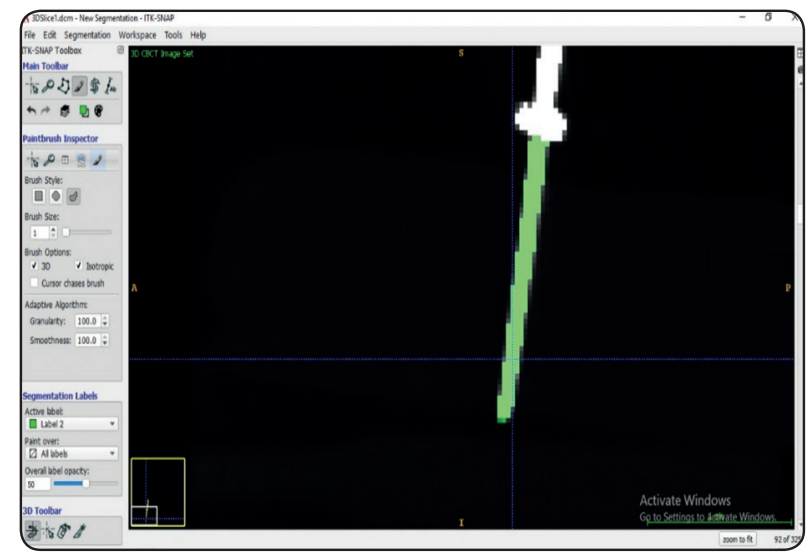

Fig. 3: Rechecking of all file pixels were included during segmentation from sagittal view at high magnification.

\section{Statistical analysis}

The collected data was organized, tabulated and statistically analysed using computer software Statistical Package for Social Science (SPSS version 21) (SPSS Inc, Chicago, IL, USA). The Kolmogorov- Smirnov was used to verify the normality of distribution of variables; Paired t-test was used for normally distributed quantitative variables, to compare between two periods. Significance of the obtained results was judged at the $5 \%$ level.

\section{RESULTS}

\section{Change of kilovoltage}

The results of the present study showed a nonsignificant difference $(p=0.688)$ between the mean of 3D radiographic volume of group $3\left(19.9 \pm 10 \mathrm{~mm}^{3}\right)$ that represent the lowest value of kilovoltage $(\mathrm{kV})$ with $50 \mathrm{KV}$ and the mean of actual volume group $\left(18.8 \pm 11.6 \mathrm{~mm}^{3}\right)$ with mean difference of $(1.1 \pm 7.7$ $\mathrm{mm}^{3}$ ) as shown in table 1 and figure 4 .

Table 1 and figure 4 showed a non-significant difference $(p=0.476)$ between the mean of $3 D$ radiographic volume of group $2\left(16.2 \pm 8.5 \mathrm{~mm}^{3}\right)$ with $60 \mathrm{KV}$ and the mean of actual volume group $\left(18.8 \pm 11.6 \mathrm{~mm}^{3}\right)$ with mean difference of $(2.6 \pm 9.6$ $\left.\mathrm{mm}^{3}\right)$.

On the other hand, a significant difference between the mean of volume measurement at group 1 with $80 \mathrm{Kv}$ and actual group as the means were $29.3 \pm 10 \mathrm{~mm}^{3}$ and $18.8 \pm 11.6 \mathrm{~mm}^{3}$ respectively, with mean difference of $10.5 \pm 5.7 \mathrm{~mm}^{3}$ as $(\mathrm{p}<0.05)$. as shown in table 1 and figure 4.

\section{- Change of voxel size:}

Table 2 and figure 5 showed a non-significant difference $(\mathrm{p}=0.508)$ between the mean of $3 \mathrm{D}$ radiographic volume of group (b) $(16.9 \pm 8.7$ $\mathrm{mm}^{3}$ ) with voxel size $180 \mu \mathrm{m}$ and the mean of actual volume group $\left(18.8 \pm 11.6 \mathrm{~mm}^{3}\right)$ with mean 
difference of $\left(1.9 \pm 7.8 \mathrm{~mm}^{3}\right)$. While, a significant difference has been showed between the mean of volume measurement at group (a) with voxel size $300 \mu \mathrm{m}$ and actual group as the means were $29.3 \pm 10 \mathrm{~mm}^{3}$ and $18.8 \pm 11.6 \mathrm{~mm}^{3}$ respectively with mean difference of $10.5 \pm 5.7 \mathrm{~mm}^{3}$.
In the present study, the results of intra-examiner measurement reliability for one selected file (file 70) and 180 voxal $4 \mathrm{~mA} 80 \mathrm{KV} 8.01 \mathrm{sec}$ showed non-significant difference between highest measure and lowest measure of $0.49 \mathrm{~mm} 3$.

Table (1): The means of 3D radiographic volume measurements and actual volume at study groups 1, 2, $3 \&$ actual.

\begin{tabular}{cllll}
\hline & Group 1 & Group 2 & Group 3 & Actual \\
\hline Min. - Max. & $11.4-40.8$ & $9.3-36.6$ & $12.2-42.5$ & $5.9-37.7$ \\
Mean \pm SD. & $29.3 \pm 10$ & $16.2 \pm 8.5$ & $19.9 \pm 10$ & $18.8 \pm 11.6$ \\
Difference from actual & $10.5 \pm 5.7$ & $-2.6 \pm 9.6$ & $1.1 \pm 7.7$ & \\
$\mathbf{p}$ & $0.001^{*}$ & 0.476 & 0.688 & \\
\hline
\end{tabular}

standard deviation $S D,{ }^{*}$ significant, - decrease, increase

Table (2): The means of 3D radiographic volume measurements and actual volume at study groups a, b \& actual.

\begin{tabular}{llll}
\hline & $\begin{array}{l}\text { Group (a) } \\
\text { 300VOXAL }\end{array}$ & $\begin{array}{l}\text { Group (a) } \\
\text { 180VOXAL }\end{array}$ & Actual \\
\hline Min. - Max. & $11.4-40.8$ & $3.6-30.4$ & $5.9-37.7$ \\
Mean \pm SD. & $29.3 \pm 10$ & $16.9 \pm 8.7$ & $18.8 \pm 11.6$ \\
Difference from actual & $10.5 \pm 5.7$ & $-1.9 \pm 7.8$ & \\
$\mathbf{p}$ & $0.001^{*}$ & 0.508 & \\
\hline
\end{tabular}

standard deviation $S D$, * significant, - decrease, increase

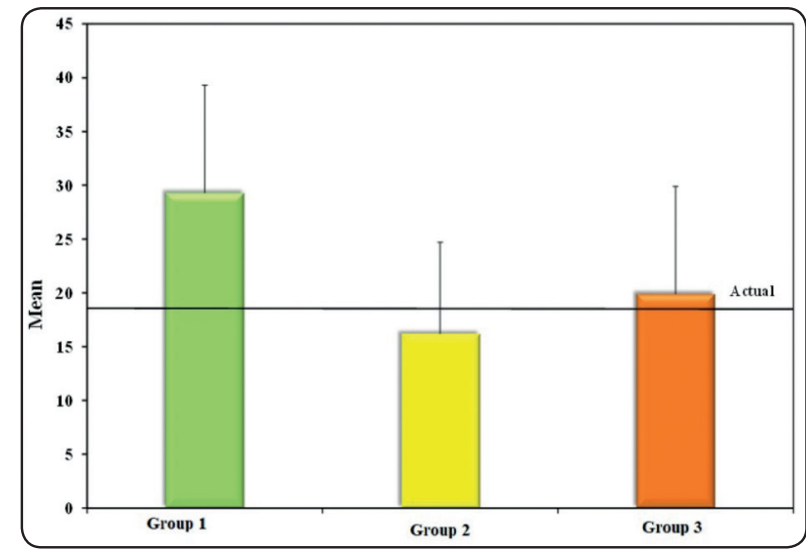

Fig. (4): The means of 3D radiographic volume measurements and actual volume at study groups 1,2, 3 \& actual.

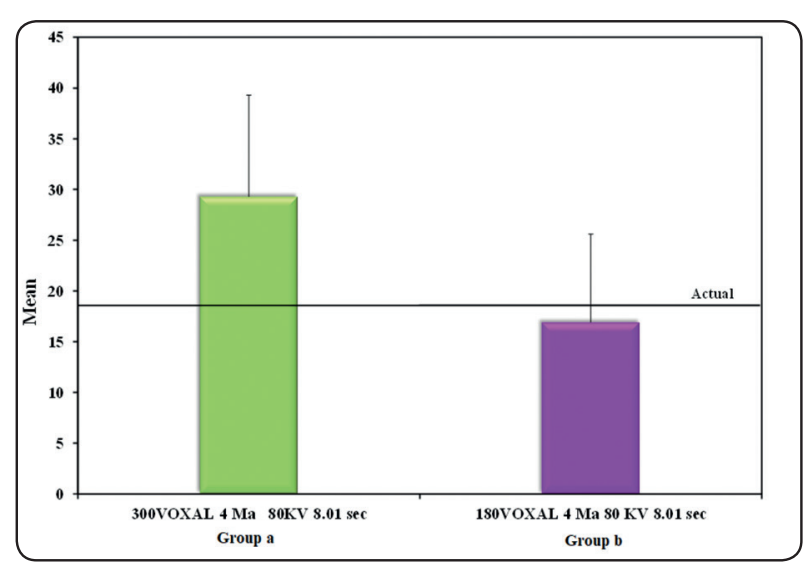

Fig. (5): The means of 3D radiographic volume measurements and actual volume at study groups a, b \& actual. 


\section{DISCUSSION}

CBCT showed very high accuracy in dental diagnosis with extremely high sensitivity and specificity results that reach $100 \%$ for both, since CBCT was used for detection and diagnosis of mandibular furcation involvement, this was reflected by comprehensive use in dental practice ${ }^{[17]}$.

Different radiographic measures can be obtained from CBCT, most of these radiographic assessments are linear measures, the transforming from linear measures to quantitative measures has a strong interest. Limited number of studies evaluated the accuracy of quantitative measures as radiographic volumetric measurement. At a study conducted by Elboraey et al., ${ }^{[17]}$ the 3D volume assessment of CBCT was evaluated. The study showed accurate and promising results for using 3D volume measurement instead of linear measurement, which may be of value especially for the reproducible assessment of bone defect topography for research purposes $^{[17]}$.

Problem of image segmentation is a very challenging problem during obtaining quantitative measures from 3D radiographic modalities as it depends mainly on sharp and highly accurate outlining of the structures of interest. Because of segmentation challenges, there is no definite strategy or algorithm for object segmentation was used by different software, some software depend on statistical modelling and machine- learning algorithms or algorithms based on warping an image to a set of expert- labelled normative atlas images ${ }^{[18]}$ or geometrically deformable models ${ }^{[19]}$.

Numerous software packages are available to analyze the 3D data acquired from CT or CBCT scan, as part of volumetric measurement process. ITK-SNAP software is a easy-use, free, with reliable package for measuring and extracting radiographic volumes, ITK-SNAP allows different modalities for identifing and outlining for the structure of interest depending on automatic segmentation or semiautomatic segmentation or self-expert outlining of the radigraphic object with the option of isotropic identification for the voxels from pixels selection, in turn these allow an accurate measurement of the volume of interest. ${ }^{[15,20]}$

In the present study, $8 \mathrm{k}$ type endodontic files were fixed at wax block paraell to each other, and then was exposed to different radiographic $\mathrm{Kv}(50$, $60,80)$ at $300 \mu$ and then divided to three groups, group $1(50 \mathrm{Kv})$, group $2(60 \mathrm{kv})$, group $3(80 \mathrm{Kv})$. After that was exposed again using two different voxel size and divided into two groups, group a (300 voxel size) and group b ( 180 voxel size), then the actual volume of the files was measured by the water displacment method.

The current study showed the following results, a non-significant difference between the mean of $3 \mathrm{D}$ radiographic volume at group $3(50 \mathrm{KV})$ and the mean of actual volume group with the same results for group $2(60 \mathrm{Kv})$ as $(\mathrm{p}>0.05)$ while at group 1 with $80 \mathrm{Kv}$ a significant difference with actual volume group, was found with mean difference of $10.5 \pm 5.7 \mathrm{~mm} 3$.

While for voxel size change, the results showed a non-significant difference between the mean of $3 \mathrm{D}$ radiographic volume of at group (b) with voxel size $180 \mu$ and actual group with mean difference of $1.9 \pm 7.8 \mathrm{~mm}^{3}$, while a significant difference between the mean of volume measurement at group (a) with voxel size $300 \mu$ and actual group with mean difference of $10.5 \pm 5.7 \mathrm{~mm}^{3}$ was found.

The results of the present study were in agreement with the result of the study conducted by Gomes et al ${ }^{[21]}$ for evaluating the accuracy of ITK-SNAP software for calculating the 3D radiographic volume using a rubber duck's head that was filled with volumes of water then radiographically exposed at fixed radiographic set. The radiographic volumes were measured using semi-automatic segmentation modality of ITK-SNAP. The results showed that ITK-SNAP is highly accurate and reliable method for radiographic volume measurement proofed by a significant relationship between ITK-SNAP 
volumes and the gold standard as $(\mathrm{p}<0.0001)$. Also, the present study showed a non-significant difference between the actual files' volumes and $3 \mathrm{D}$ radiographic volumes in groups 2, 3 and $\mathrm{b}$ using ITK-SNAP software.

On the other hand, Sang et al. ${ }^{[1]}$ found that decreasing voxel size from 300 to $150 \mu \mathrm{m}$, did not improve the accuracy of linear, volumetric, and geometric 3D tooth reconstruction from CBCT, which is disagreed with the results of the present study as increasing the voxel from .30 to $.18 \mathrm{~mm}$ lead to more accurate $3 \mathrm{D}$ volume measurements since the results from comparing the $3 \mathrm{D}$ radiographic volume for group (a) with voxel size $0.3 \mathrm{~mm}$ with the actual volume converted from significant difference to non-significant difference for group (b) with voxel size of $.18 \mathrm{~mm}$ with means volume difference of $10.5 \pm 5.7 \mathrm{~mm}^{3}$ and $1.9 \pm 7.8 \mathrm{~mm}^{3}$ respectively.

The volume change between 3D radiographic volumetric measurement and the actual volume of the scanned object mainly because of different radiographic artefacts that affect the quality of CBCT images $^{[22]}$, as, beam hardening effect, extinction artefact, motion artefact, ring artefact, aliasing artefact, noise-scatter artefact, partial volume artefact and finally voxel size that reduce the accuracy with increase of voxel size. [23]

The theory in usage of average CT value for voxel size on the CT images, does not reflect the actual CT value of scanned different structures, which is known as the partial-volume effect that is a common artefact of $\mathrm{CT}^{[22]}$. Therefore, when a larger voxel size was used, the reconstructed volume was larger than its reality by artefacts this might be an explanation for different results when changing the voxel size, which is confined with a study conducted Dong et al., since that with increased voxel size, the artefacts of CBCT scanning increased from $.125 \mathrm{~mm}$ to $.40 \mathrm{~mm}$, and the volumetric measurement increased for each corresponding Hounsfield (HU) threshold of segmentation ${ }^{[9]}$.
In the current study, the different results with changing kv from 80 t0 60 to $50 \mathrm{Kv}$ as in groups 1 , 2 and 3 respectively, as with group 2 and 3 , there are non-significant difference with the actual volume group with best result for group 3 that have the least $\mathrm{Kv}$ of $50 \mathrm{Kv}$ while for group 1 with the highest $\mathrm{Kv}$ of $80 \mathrm{Kv}$, the results showed a significant difference with the actual volume group. These results can be explained with that the increase of $\mathrm{Kv}$ lead to increase of metal artifact, compromising 3D volume measurement and reconstruction. On the other hand, decreasing the voxel size from $300 \mu$ to $180 \mu$ lead to non-significant difference between group (b) with $180 \mu$ voxel and actual volume group reflecting the importance of decreasing voxel size for better 3D volume measurement and reconstruction and for significantly reduction of metal artifact reflected by significant difference between group (a) and (b) with the same high kilovoltage of $80 \mathrm{Kv}$ for both groups. ${ }^{[22,24]}$.

Upon the results of this study, it can be concluded that, both change in kilovoltage and voxel size parameters affect significantly the $3 \mathrm{D}$ volume measurement and reconstruction, ITK-SNAP software is high accurate for 3D volume measurement for further clinical and radiographic studies and highly recommended from the author for 3D radiographic volume measurement, replacing conventional linear measurement for different clinical studies as periodontal defect measurement, peri-implant defect assessment, forensic dentistry analysis and pedodontics and orthodontics analysis. It also recommended that for accurate 3D volume measurement, it is better to decrease $\mathrm{Kv}$ and voxel size as much as possible specially when a metallic object as the implant will be used in the study work.

Fixed and highly calibration has been done to the CBCT in this study for all the study groups, the CBCT images were with high and accurate quality and with well-organized study, therefore, the results of this study can provide some reference to guide this kind of $\mathrm{CBCT}$ reconstruction and $3 \mathrm{D}$ volume measurement studies. 


\section{ACKNOWLEDGEMENT}

The author is thankful to Prof. Dr. Yasser Makaky, professor of oral radiology, Tanta University, Egypt, for his valuable help and effort, as well as to Marwan Ahmed Alqurashi, dental student, Taibah University, Kingdom of Saudi Arabia, for his help during measuring the actual volume during the study and for his valuable effort.

\section{REFERENCES}

1. White, S.C. and M.J. Pharoah, White and Pharoah's Oral Radiology E-Book: Principles and Interpretation. 2018: Elsevier Health Sciences.

2. Dawood, A., S. Patel, and J. Brown, Cone beam CT in dental practice. British dental journal, 2009. 207(1): p. 23-28.

3. Scarfe, W. and A. Farman, Soft tissue calcifications in the neck: Maxillofacial CBCT presentation and significance. AADMRT Currents, 2010. 2(2): p. 3-15.

4. Ashraf, A.B., et al., A multichannel Markov random field framework for tumor segmentation with an application to classification of gene expression-based breast cancer recurrence risk. IEEE transactions on medical imaging, 2012. 32(4): p. 637-648.

5. Lee, R.J., et al., Three-dimensional monitoring of root movement during orthodontic treatment. American Journal of Orthodontics and Dentofacial Orthopedics, 2015. 147(1): p. 132-142.

6. Mangano, F.G., et al., Custom-made, root-analogue direct laser metal forming implant: a case report. Lasers in medical science, 2012. 27(6): p. 1241-1245.

7. Shen, P., et al., Accuracy evaluation of computer-designed surgical guide template in oral implantology. Journal of Cranio-Maxillofacial Surgery, 2015. 43(10): p. 2189-2194.

8. Eggers, G., J. Mühling, and C. Hofele, Clinical use of navigation based on cone-beam computer tomography in maxillofacial surgery. British Journal of Oral and Maxillofacial Surgery, 2009. 47(6): p. 450-454.

9. Dong, T., et al., Accuracy of in vitro mandibular volumetric measurements from CBCT of different voxel sizes with different segmentation threshold settings. BMC oral health, 2019. 19(1): p. 206.

10. Kamburoglu, K., et al., Comparative assessment of subjective image quality of cross-sectional cone-beam computed tomography scans. Journal of Oral Science, 2011. 53(4): p. 501-508.

11. Sang, Y.-H., et al., Accuracy assessment of three-dimensional surface reconstructions of in vivo teeth from conebeam computed tomography. Chinese medical journal, 2016. 129(12): p. 1464.

12. Spin-Neto, R., E. Gotfredsen, and A. Wenzel, Impact of voxel size variation on CBCT-based diagnostic outcome in dentistry: a systematic review. Journal of digital imaging, 2013. 26(4): p. 813-820.

13. Karatas, O.H. and E. Toy, Three-dimensional imaging techniques: A literature review. European journal of dentistry, 2014. 8(1): p. 132.

14. Ye, N., F. Jian, and W. Lai, Effect of voxel size and partial volume effect on accuracy of tooth volumetric measurements with cone beam CT. Dentomaxillofacial Radiology, 2013.42(5).

15. Almuzian, M., H.M. Al Ghatam, and L. Al-Muzian, Assessing the validity of ITK-SNAP software package in measuring the volume of upper airway spaces secondary to rapid maxillary expansion. journal of orthodontic science, 2018. 7.

16. Robert, U.W., S.E. Etuk, and O.E. Agbasi, Modified water displacement method and its use for determination of bulk density of porous materials. Journal of Renewable Energy and Mechanics, 2019.2(01).

17. Momamed Omar Elboraey, H.I.S., Samia A. Darwish, Mona Yehia Abd El Razzak The Effectiveness of Chitosan Collagen Membrane with and without Hydroxyapatite Particles in the Treatment of Class II Mandibular Furcation Defects in Humans: A Randomized Clinical and Radiographic Evaluation. EGYPTIAN DENTAL JOURNAL, 2018. 4(july): p. 160-175.

18. Iglesias, J.E. and M.R. Sabuncu, Multi-atlas segmentation of biomedical images: a survey. Medical image analysis, 2015. 24(1): p. 205-219.

19. Heimann, T. and H.-P. Meinzer, Statistical shape models for 3D medical image segmentation: a review. Medical image analysis, 2009. 13(4): p. 543-563.

20. El, H. and J.M. Palomo, Measuring the airway in 3 dimensions: a reliability and accuracy study. American Journal of Orthodontics and Dentofacial Orthopedics, 2010. 137(4): p. S50. e1-S50. e9.

21. Gomes, A.F., et al., Accuracy of ITK-SNAP software for 3D analysis of a non-regular topography structure. Oral Radiology, 2019: p. 1-7.

22. Nagarajappa, A.K., N. Dwivedi, and R. Tiwari, Artifacts: The downturn of CBCT image. Journal of International Society of Preventive \& Community Dentistry, 2015. 5(6): p. 440.

23. Hassan, B., et al., Influence of scanning and reconstruction parameters on quality of three-dimensional surface models of the dental arches from cone beam computed tomography. Clinical oral investigations, 2010. 14(3): p. 303-310.

24. Schulze, R., et al., Artefacts in CBCT: a review. Dentomaxillofacial Radiology, 2011. 40(5): p. 265-273. 